\title{
A large abdominal desmoid tumour associated with pregnancy and puerperium
}

\author{
Setu Rathod*, Sunil Kumar Samal, Purna Chandra Mahapatra
}

Department of Obstetrics \& Gynaecology, SCB Medical College, Cuttack, Odisha, India

Received: 20 January 2014

Accepted: 02 February 2014

\author{
*Correspondence: \\ Dr. Setu Rathod, \\ E-mail: seturathod@gmail.com
}

(C) 2014 Rathod S et al. This is an open-access article distributed under the terms of the Creative Commons Attribution Non-Commercial License, which permits unrestricted non-commercial use, distribution, and reproduction in any medium, provided the original work is properly cited.

\begin{abstract}
We report a rare case of huge abdominal desmoid tumour first detected during pregnancy. The patient delivered vaginally and the size of the tumour increased during puerperium for which resection was done. Most of these tumours occur in the abdominal muscles particularly right rectus abdominis, perhaps related to trauma from abdominal stretching and movement. These tumours are known to regress spontaneously after delivery which was not in our case. Subsequent pregnancies do not appear to result in recurrence in either FAP (Familial Adenomatous Polyposis) or non-FAP patients. It is not clear from currently available data whether pregnancy associated desmoids are molecularly distinct from other desmoids.
\end{abstract}

Keywords: Abdominal wall, Desmoid, Fibromatosis, Pregnancy, Puerperium

\section{INTRODUCTION}

Desmoid tumor also known as aggressive fibromatosis or musculo-aponeurotic fibromatosis is a monoclonal fibroblastic proliferation arising in musculoaponeurotic structures. Desmoid tumors are rare, benign tumors with locally aggressive behavior more common in young women. The incidence of sporadic desmoid tumor is estimated to be 2-4 per million individuals per year. ${ }^{1}$ Desmoid tumors are classified according to their location into extra abdominal and abdominal type. The most common sites are the shoulder girdles and rectal muscles. ${ }^{2}$ The causes of desmoid tumor are uncertain and may be related to operation, trauma, hormonal and genetic factors. This case report shows a rare occurrence of a huge abdominal desmoid tumour during pregnancy and further increasing in size during puerperium.

\section{CASE REPORT}

A 22 year Gravida 2 Para 1 attended our OPD at 39 weeks gestational age with a lump of size $25 \times 24 \mathrm{~cm}$ in lower abdomen first noticed at 12 weeks gestation, progressively increasing in size throughout pregnancy. General and obstetric examination revealed no abnormality. There was no relevant family history. She had normal vaginal delivery. During puerperium the tumour grew rapidly to a size of 32 x $30 \mathrm{cms}$. She developed pain abdomen and an ulcer of size $5 \times 5 \mathrm{~cm}$ on the skin overlying the tumour. On physical examination, the tumor was hard in consistency, well defined arising from the parietal wall of abdomen. At 6 weeks puerperium, USG report showed a huge hypoechoic mass of size $30 \times 28 \mathrm{~cm}$ involving the lower abdomen and FNAC revealed clusters of ovoid to spindleloid fibroblasts with bipolar cytoplasmic processes and fragments of collagenous matrix suggestive of desmoid. The mass was excised surgically. A diamond shaped incision was given around the tumor and the mass was enucleated by separating the connective tissue and abdomen closed longitudinally. Post-operative period was uneventful. At follow up after one month she had no complaints and examination revealed no abnormality. The histopathological report confirmed the diagnosis to be desmoid with negative margins. 


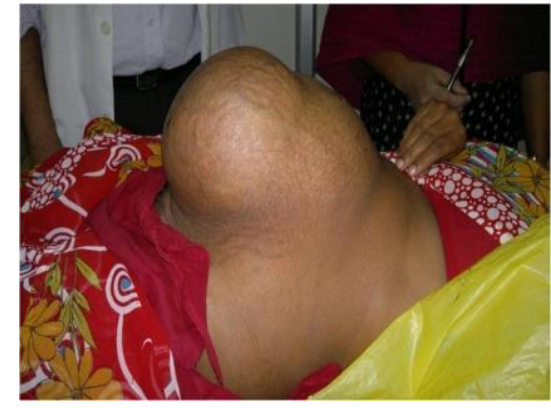

Figure 1: During puerperium the tumour grew rapidly to a size of $32 \times 30 \mathrm{cms}$.

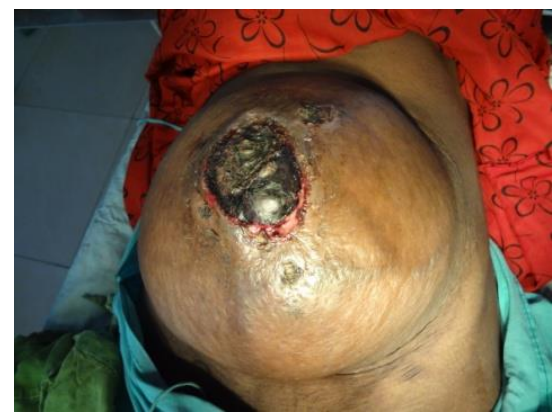

Figure 2: Ulcer of size $5 \times 5 \mathrm{~cm}$ on the skin overlying the tumour.

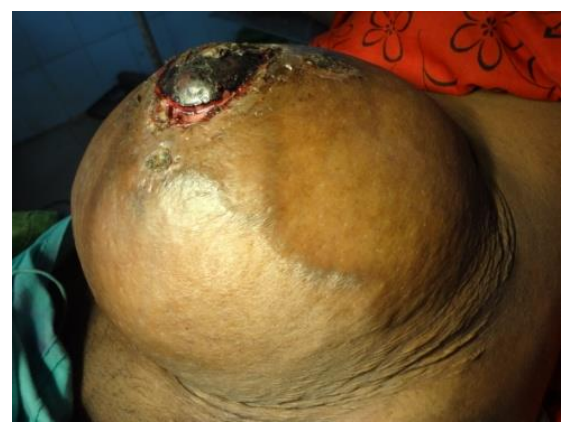

Figure 3: At 6 weeks puerperium, USG report showed a huge hypoechoic mass of size $30 \times 28 \mathrm{~cm}$ involving the lower abdomen.

\section{DISCUSSION}

Pregnancy associated desmoid tumours have been a subject of interest since the first description by Mcfarlene in $1832 .^{3}$ Desmoid tumours account for $0.03 \%$ of all neoplasms and $<3 \%$ of all soft tissue tumours but the incidence in the Familial Adenomatous Polyposis (FAP) patient ranges from $3.4-34 \%$. Majority of tumours are found in women of age 20-40 years. In contrast to sporadic cases, FAP associated desmoids (Gardner's syndrome) are mainly intra-abdominal type.

Desmoid tumours often develop during, after pregnancy or following uptake of contraceptive pills. It is well known that desmoids may be associated with trauma of different sorts. Others have attempted to link hormonal and immune system changes that occur in pregnancy with desmoids development. The possible role of these factors is suggested by the description of spontaneous desmoids regression after delivery. ${ }^{4}$ Further the possible role of estrogen is suggested by the occasional report of desmoids tumours responding to treatment with the antiestrogen, tamoxifen.

Typically, desmoids arising during or shortly after pregnancy are almost always located in the abdominal wall. Single cases of desmoid tumour arising in the vulva, larynx, neck and popliteal space during pregnancy have been described, while only a few cases of pregnancyassociated intra-abdominal desmoids, originating from the retroperitoneum, mesentery and pelvis have been reported.

In the study by Robinson et al. ${ }^{6}$ a case series of ten patients with pregnancy associated desmoids tumours were studied. The tumours ranged in size from $2 \mathrm{~cm}$ to 16 $\mathrm{cm}$ with an average size of $4.5 \mathrm{~cm}$. Out of 10 patients, only 3 delivered vaginally. Although only a few cases have been reported, desmoid tumour should be included in the differential diagnosis of intra-abdominal tumours detected during or shortly after pregnancy. The enormous tumour size in the present case is noteworthy. In one report, a pelvic desmoid tumour interfered both with normal maturation and delivery of the fetus as well as the patient's ability to void and defecate, prompting surgical intervention at 23 weeks of gestation. Following the resection the patient delivered a healthy full-term baby. ${ }^{7}$ In the other pelvic desmoid case, the tumour obstructed labour, necessitating a Caesarean section and subsequent tumour excision. ${ }^{8}$

Imaging study (CT and MRI) may delineate the tumor sites; determine their extent, relationship with adjacent organs and vital structures. ${ }^{9}$ Spontaneous regression is reported to occur after menopause, oophorectomy and after anti-estrogen treatments. The large soft tissue defect after resection can be achieved by direct repair or using synthetic materials (Mesh).The other modality of treatment is antihormonal drugs, NSAIDS and cytotoxic drugs such as doxorubicin, dacarbazine which can be considered for symptomatic, unresectable, clinically aggressive desmoid tumor not responding to conventional treatment. $^{10}$

Subsequent pregnancy is not necessarily a risk factor for recurrent or new desmoid tumour. Based on the median time to eventual desmoid recurrence after resection, it may be advisable for the patient to allow for a 2-year follow-up period before planning an eventual subsequent pregnancy. 5

\section{CONCLUSION}

Inspite of the huge size of desmoid tumour associated with pregnancy, she delivered at term vaginally and was operated at 6 weeks of puerperium without any complications. 
Funding: No funding sources

Conflict of interest: None declared

Ethical approval: Not required

\section{REFERENCES}

1. Bernardina Rampone, Corrado Pedrazzani, Daniele Marrelli, Enrico Pinto, Franco Roviello. Updates on abdominal desmoids tumours. World J Gastroenterol. 2007 December;13(45):5985-8.

2. Vichit Viriyaroj, Narongchai Yingsakmongkol et al. A Large Abdominal Desmoid Tumour Associated with Pregnancy. J Med Assoc Thai. 2009;92(Suppl 3):S72-5.

3. Macfarlane J. Clinical Reports of the Surgical Practice of the Glasgow Royal Infirmary. Glasgow, UK: David Robertson; 1832: xvi, 314.

4. Rocha F., George S., Fletcher C., Bertagnolli M., Butrynski J., Demetri G., Morgan J., Wagner A., Raut C. Pregnancy-associated desmoid tumors: A distinct clinical entity. Ann Surg Oncol. 2008;15:66.

5. Eelco de Bree, Eustathios Dimitriadis, Elpida Giannikaki, Evangelia G. Chryssou, John Melissas. A giant pregnancy-associated intra-abdominal desmoid tumour: not necessarily a contraindication for subsequent pregnancy. World Journal of Surgical Oncology. 2013;11:277.

6. William A. Robinson, Colette McMillan, Amy Kendall, Nathan Pearlman. Desmoid Tumors in Pregnant and Postpartum Women. Cancers. 2012;4:184-92.

7. Firoozmand E, Prager E. Pelvic desmoid tumor: threat to mother and fetus. Am Surg. 2001;67:12135 .

8. Tankshali R, Srivastava S, Anshuman K, Shah M. An unusual presentation of aggressive fibromatosis (retroperitoneal desmoid tumour) in pelvic cavity presented as obstructed labour. J Indian Med Assoc. 2011;109:589-91.

9. Sakorafas GH, Nissotakis C, Peros G. Abdominal desmoids tumours. Surg Oncol. 2007;16:131-42.

10. Kulaylat MN, Karakousis CP, Keaney CM, Mc Corvey D, Bem J, Ambrus Sr JL. Desmoid tumour: a pleomorphic lesion. Eur J Surg Oncol. 1999;25:48797.

DOI: $10.5455 / 2320-1770 . i j r \operatorname{cog} 20140360$

Cite this article as: Rathod S, Samal SK,

Mahapatra PC. A large abdominal desmoid tumour associated with pregnancy and puerperium. Int $\mathrm{J}$ Reprod Contracept Obstet Gynecol 2014;3:270-2. 